\title{
Framework urges physicians to proceed with caution on palliative sedation
}

$\mathrm{E}$ asily the most controversial tool in the palliative care arsenal, the practice of sedating patients near death to relieve intolerable and refractory suffering may also be the most widely misunderstood.

Debate over the therapy's potential for misuse, confounded by a lack of clear or consistent guidance on its ethical and medically appropriate use, has misled the public and even some health professionals to view it as a covert form of euthanasia, says Dr. Larry Librach, director of the Temmy Latner Centre for Palliative Care at Mount Sinai Hospital in Toronto, Ontario. "In these days of budget cutbacks, people are suspicious we just want to speed up death to free up resources."

Part of an expert team drafting a national framework to better define and guide the use of continuous palliative sedation therapy in Canada, Librach says confusion over when and how to administer the therapy has only served to feed those fears.

"Some physicians aren't aware of the indications to start treatment, they don't know how to conduct an appropriate assessment, so they make a wrong diagnosis, they don't consult the patient or their family and they end up sedating someone with months left to live," he explains. "Leave a person in it for a week without food or fluid and they'll die prematurely."

Without clear guidance, physicians may also prescribe the wrong drugs to achieve sedation, such as opioids, or may not monitor the patient closely enough to ensure they're properly sedated, says Dr. Doreen Oneschuk, a palliative care physician at Grey Nuns Hospital in Edmonton, Alberta, and a member of the team drafting the framework. "Use the wrong drugs and the patient can end up more agitated or can develop other distressing side effects, like muscle twitches. You also want to make sure you're titrating fairly quickly because you don't want to leave someone dis-

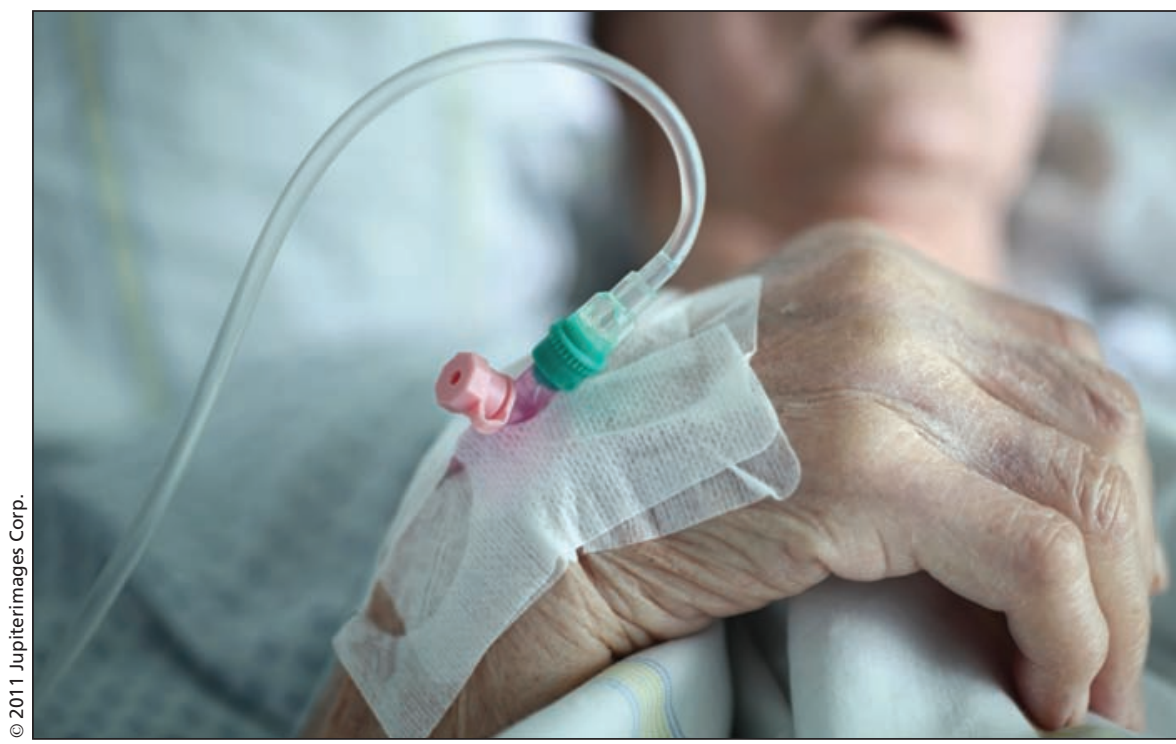

A draft policy framework proposes that continuous palliative sedation therapy only be administered in the last two weeks of life, as a final resort when all other efforts to treat a patient's suffering have been exhausted and it's unlikely they will recover to a former level of functioning.

tressed for hours or administer too much and suppress their respiration."

Canadian protocols for administering continuous palliative sedation therapy are largely inconsistent, where such policies exist, says Librach. "Some institutions have guidelines that just focus on which drug to use, some have instructions on how to talk to patients and families, and others have nothing at all. For example, we have a large palliative care program here at Mount Sinai and yet there's nothing written about this process."

The absence of a clear definition for continuous palliative sedation therapy — known more ambiguously as "terminal sedation" or "continuous deep sedation" - has also made it difficult to differentiate the legitimate therapy from forms of euthanasia. In Quebec last year, $81 \%$ of medical specialists surveyed said they had seen "euthanasia" practised, and $48 \%$ said that palliative sedation "can be likened to a form of euthanasia" (www.fmsq.org/mag electronique_1009/e/actualites.html).

The draft framework proposes con- tinuous palliative sedation therapy only be administered in the last two weeks of life, as a final resort when all other efforts to treat a patient's suffering have been exhausted and it's unlikely they will recover to a former level of functioning.

Limiting the therapy to the last two weeks of life removes the question of whether it may hasten death, says Librach. "With or without hydration or nutrition, the patient's trajectory remains the same."

While the calculation of how long a patient has left to live is more an art than a science, there are often "signposts" to indicate when a patient is rapidly deteriorating, including "weakness increasing day by day or a sudden drop in hunger, thirst or consciousness."

The draft framework also calls for careful monitoring of the patient, as well as follow up with family and staff, to ensure sedation continues to be appropriate to treat a patient's suffering up until their death.

"If the patient seems stable, it's then a question of do we continue treatment 
or lighten it up to get a better idea of where the patient's at," says Librach.

Caution is particularly necessary when assessing a patient for continuous palliative sedation therapy to treat nonphysical suffering, says Blair Henry, a clinical ethicist at Sunnybrook Health Sciences Center in Toronto, Ontario, and a member of the team drafting the framework. "It's rare that such suffering would be called intolerable and refractory, but it's part of everyone's practice experience that you do occasionally meet people who are in such distress or crisis that no intervention of a psychologist or chaplain or any team member can relieve."

While such cases are most com- monly confused for physician-assisted suicide, withholding treatment, as a rule, would not be appropriate, says Librach. "I find it very difficult to separate out. In one case, I knew my patient was going to die a very difficult death with his bowel perforating but up to that point he wouldn't have much pain. He told me he was suffering waiting for the end, and I told him we would sedate him when the time came. We never were able to control his pain at end-oflife. He has this acute event, no one was standing by his bedside at the ready, and I've felt guilty ever since because he died a quick but very horrible, painful death."
Although patients regularly "walk away from the treatment table," refusing chemotherapy or dialysis, a patient's ability to "walk away from their sentience" is decidedly more limited by societal pressures, says Henry. "We're holding people from therapy they may in fact need because of value judgments we've made that a person should find meaning in their suffering."

The draft framework will be submitted to the Canadian Society of Palliative Care Physicians for review in the coming months. A final version will be published in 2011. — Lauren Vogel, CMAJ

CMAJ 2011. DOI:10.1503/cmaj.109-3766

\section{Infectious risks in family doctors' offices}

$\mathrm{W}$

hat a difference a year makes. During pandemic (H1N1) 2009, primary care providers were scurrying around offices and clinics removing books and toys from waiting rooms, positioning bottles of hand sanitizer, passing out surgical masks to patients who presented with a cough or fever and using hospital-grade disinfectant to mop up floors when a suspected H1N1 case left their office or clinic.

They were, of course, following infection control guidelines established and recommended by the College of Family Physicians (CFPC) and a number of other groups during the height of the pandemic.

As the threat fades into memory, there's an understandable tendency to hide the mops in the closet and leave the masks in the box.

But Ontario's top infectious disease doctor is urging primary care providers to maintain aggressive infection prevention and control measures in their practices year-round.

Returning to business-as-usual would be a mistake, says Dr. Doug Sider, acting director of infectious disease prevention and control for the Ontario Agency for Health Protection and Promotion. "To recommend that we only consider more aggressive compliance with all of these infection prevention and control measures in the face of something like pandemic influenza, and then step back, relax our vigilance, relax

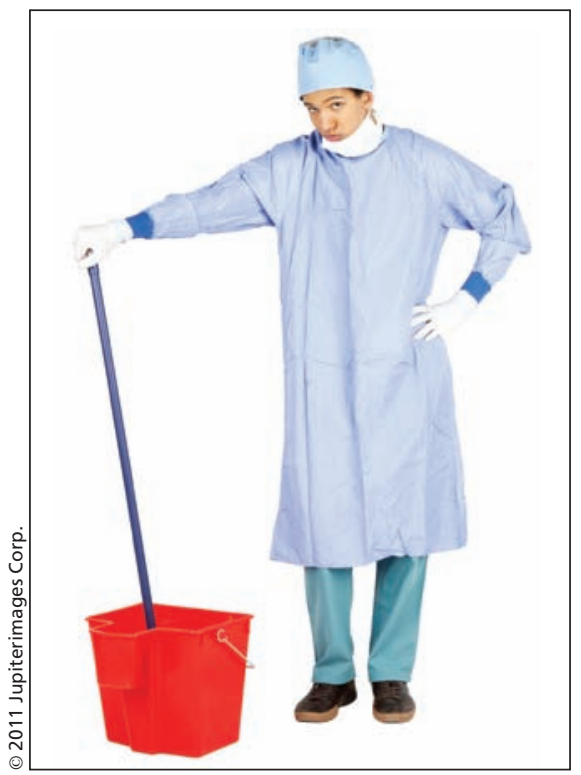

Keeping the mops moving is good infection control for practice, even though the public health threat posed by pandemic (H1N1) 2009 has abated.

our expectations, relax our urging to compliance at other times, just doesn't make a lot of sense to me."

The CFPC has never developed infection prevention and control guidelines for standard family practice. Although it was one of the associations responsible for developing and issuing the guidelines during the pandemic (along with the Canadian Medical Association, the National Specialty Society for Community Medicine and the Canadian Public Health Association), those do not apply outside of a pandemic, says Jayne Johnston, the college's communications manager.

The college doesn't have a general infection control policy telling members how they should, or should not, run their practices on a day-to-basis, Johnston says.

But some of those recommendations would be good practice year-round, particularly as the flu season descends, says Sider, who suggests that primary care doctors always ensure staff is immunized with the seasonal flu vaccine and that standardized procedures be adopted for hand hygiene and the conditions in which masks should be used.

Sider also urges regular screening for febrile respiratory illness and depending on office design, the adoption of measures to contain its spread, such as asking patients who present with a cough and fever to sit in one part of the waiting room. As an alternative, staff can use a telephone triage system to screen for respiratory illness and then cluster those appointments near the end of the day, he adds. That would limit transmission and allow for the office to be sanitized properly before the next business day.

Such measures are a challenge for many family practices, Sider acknowledges. "It's easy to say, but it's really hard to put into place. Hospitals have environmental services people that can 